\title{
Optimization of a Pheromone Lure for Spodoptera frugiperda (Smith) in Central America
}

\author{
Romano Andrade ${ }^{a}$, Carlos Rodriguez, and Allan C. Oehlschlager ${ }^{a^{*}}$ \\ ${ }^{a}$ ChemTica Internacional, S. A., Apdo. 159-2150, San Jose, Costa Rica \\ ${ }^{\mathrm{b}}$ DelMonte Fresh Produce, Pavas, Costa Rica
}

\begin{abstract}
A captura em massa da Spodoptera frugiperda (Smith) na América Central requer um atrativo altamente eficiente e com consistência na taxa de captura. Atrativos empregados para S. frugiperda provenientes da América do Norte e Inglaterra apresentaram taxas de captura irregulares quando aplicados no campo. A reinvestigação de quatro acetatos (Z9-14Ac, Z11-16Ac, Z7-12Ac e Z9$12 \mathrm{Ac}$ ) para $S$. frugiperda encontrada na Costa Rica revelou que Z7-12Ac e Z9-12Ac, quando empregados de maneira isolada, se mostraram altamente atraentes. Combinação binária de Z7-12Ac e Z9-14Ac aumentou consideravelmente o poder de atração sendo, no mínimo, dez vezes mais atraentes à S. frugiperda da Costa Rica que os atrativos provenietes da América do Norte e Inglaterra. $\mathrm{A}$ adição de Z11-16Ac à combinações binárias de Z9-14Ac e Z7-12Ac , causou um pequeno aumento nas taxas de captura. Quando a concentração de $Z 7-12 \mathrm{Ac}$ é aumentada para $5 \%$ em atrativos contendo Z9-14Ac, Z11-16Ac e Z7-12Ac as taxas de captura diminuem significativamente. O atrativo otimizado contém $Z 7-12$ Ac que é um composto não descrito anteriormente para $S$. frugiperda da região caribenha.
\end{abstract}

Mass trapping of Spodoptera frugiperda (Smith) in Central America required a high performance lure with consistent capture rates. S. frugiperda lures from North America and England gave erratic capture rates under field conditions. A reinvestigation of four acetate attractants (Z9-14Ac, Z11$16 \mathrm{Ac}, \mathrm{Z7}-12 \mathrm{Ac}$ and Z9-12Ac) for S. frugiperda present in Costa Rica revealed that Z7-12Ac and Z9-12Ac were highly attractive to $S$. frugiperda when presented alone. Binary combination of Z7$12 \mathrm{Ac}$ with Z9-14Ac significantly increased attraction and was at least 10 times more attractive to $S$. frugiperda in Costa Rica than North American or English lures. Addition of Z11-16Ac to binary combinations of Z9-14Ac and Z7-12Ac marginally increased capture rates. If the concentration of $\mathrm{Z7}-12 \mathrm{Ac}$ is increased to $5 \%$ in lures containing Z9-14Ac, Z11-16Ac and Z7-12Ac capture rates significantly decrease. The optimized lure contains $\mathrm{Z7}-12 \mathrm{Ac}$ that is a component not previously reported in $S$. frugiperda from the Caribbean region.

Keywords: Spodoptera frugiperda, pheromone blend, trapping

\section{Introduction}

Spodoptera frugiperda is a major pest of many crops in the Americas ${ }^{1}$. Management is most often by application of chemical insecticides or biologicals such as Bacillus thuringiensis. The female-produced sex pheromone of $S$. frugiperda is often used to monitor populations to time insecticide application ${ }^{2,3}$. Additional pheromone-based management strategies include disruption of mating and mass trapping 4,5 . The explosive population growth of $S$. frugiperda and $S$. sunia during the two month crop cycle of watermelon and cantaloupes make these important pests of these crops in Costa Rica ${ }^{6}$. The high temperatures and windy conditions in the region in which melons are grown make

*e-mail: info@mail.pheroshop.com use of mating disruption techniques for management of Spodoptera species especially challenging. Success of mass trapping of Lepidoptera has been achieved only under low population conditions 4,5 . Melon pest managers in Costa Rica began mass trapping $S$. sunia and $S$. frugiperda in 1992 as a means to maintain initial low populations and reduce expensive applications of Bacillus thuringiensis. Since 1992 mass trapping (4-5 traps per hectare) has been practiced in $>2.000$ hectares of melons to help manage populations of both Spodoptera species. This technique has routinely lowered by $30-70 \%$ the number of Bacillus thuringiensis applications required to maintain larval damage at economically acceptable levels*. While pheromone lures for $S$. sunia consistently gave high

* C. Rodriguez, unpublished results. 
performance under field conditions ${ }^{6}$ lures for $S$. frugiperda gave erratic field results. This study was prompted by the inability of $S$. frugiperda lures from North American and European sources to attract significant numbers of $S$. frugiperda to universal moth traps used in operational mass trapping programs.

Failure of pheromone lures constructed to attract $S$. frugiperda in North America to attract this species in Costa Rica might be understood in terms of population isolation. The high migratory ability of $S$. frugiperda in North America would be expected to maintain homogeneous populations within North America ${ }^{7}$. A study of population fluctuation of $S$. frugiperda from the French Guyana to Canada suggested migration from the Caribbean region to North America was not significant ${ }^{8}$.

The pheromone gland of North American $S$. frugiperda is reported to contain Z9-14Ac : Z9-14Al : Z11-16Ac : Z712Ac : Z11-16Al : Z11-12Ac : 12Ac : Z9-12Ac in a ratio of $63: 13: 9: 4: 3: 2:$ Trace $:$ Trace ${ }^{9}$. The effluvium consisted of Z9-14Ac : Z11-16Ac : Z7-12Ac : Z11-12Ac : 12Ac in the ratio of $90: 3: 3: 2: 2^{9}$. Female pheromone gland extracts of $S$. frugiperda from Guadaloupe are reported to contain Z9-14Ac : Z11-16Ac : Z9-12Ac in a ratio of $7: 1: 2$ (cited as a personal communication from C. Descoins $)^{10}$.

Field trapping results in North America demonstrated that lures containing Z9-14Ac or Z9-12Ac alone were attractive $^{11}$. Addition of Z,E,9,12-14Ac and Z7-12Ac or E9-12Ac to Z9-12Ac decreased capture rates ${ }^{11,12}$ while addition of 2\% Z11-16Ac or Z9-14Ac to Z9-12Ac did not alter capture rates ${ }^{10}$. Superior attraction was reported to lures containing Z9-14Ac and Z7-12Ac in a ratio of 99.4 : 0.6 and to the five acetates in the effluvium of $S$. frugiperda from North America at their natural ratios ${ }^{9}$.

Given erratic capture rates of lures constructed to capture $S$. frugiperda in North America and our requirement for consistently high capture rates we investigated the composition of existing temporate zone lures and reexamined attraction of $S$. frugiperda in Costa Rica to blends of Z9-14Ac, Z11-16Ac, Z7-12Ac and Z9-12Ac which are the four acetates most commonly associated with attraction from previous studies.

\section{Experimental}

Chemicals: Pheromone components were used as purchased from Bedoukain Research, 21 Finance Drive, Danbury Conn., USA.

Extraction of commercial lures: Commercial lures for $S$. frugiperda (2 from each source, Trece, Inc., USA and Agrisense, England) were separately extracted by placing each lure in a glass vial with $2 \mathrm{~mL}$ of analytical grade hexane for 48 hrs. After this time a known amount of tetradecane internal standard was added and an aliquot removed by syringe for gc analysis. Identities of eluting components were confirmed by co-elution with known standards.

Analytical Procedures: Purity of pheromone components and analysis of commercial lures was conducted by gas chromatography and gas chromatography/mass spectrometry. In the case of purchased pheromone components analyses revealed each component was $>95 \%$ pure. In the case of Z9-12Ac we confirmed that E9-12Ac which is an inhibitor was present in less than $1 \%$.

GC analysis were conducted on a HP 5890 gas chromatograph using a 30 meter DB-5 fused silica column $0.25 \mathrm{~mm}$ ID (0.25 mm film thickness) with FID detection. GC/MS analysis was conducted on a HP 5973 mass detector coupled to a HP6890 gas chromatograph using the same column.

Lures: Commercial lures from North America and England were septa and capsules respectively and contained $\sim 1.6 \mathrm{mg}$ pheromone blend. Lures produced in Costa Rica consisted of membrane release devices containing 1-2\% solutions of test pheromones in a proprietary carrier. Lures produced in Costa Rica contained $2 \mathrm{mg}$ of pheromone blend.

Field Experiments: Traps used were green Universal Moth Traps manufactured in Costa Rica and containing water or DDVP as the killing agent. Traps were placed on iron bars just above the canopy in cantaloupe or rice plantations in Guanacaste or the Central Pacific Coast of Costa Rica. Traps were at least 20 meters apart and 20 meters from any border. Complete randomized block design was used for all experiments.

Statistics: Captures were analyzed for normal distribution and where necessary transformed as indicated to achieve homogeniety. ANOVA (fully factorial routine) was conducted using Systat 5.2.1 with subsequent analysis of means by Bonferonni t-test comparison. Within the same experiment means followed by a different letter are significantly different by Bonferonni t-test, $\mathrm{P}>0.95$.

\section{Results and Discussion}

Based on reported trapping studies it was expected that commercial lures for $S$. frugiperda from North America and England would contain Z9-14Ac and Z7-12Ac or all five acetates found in the effluvium found from $S$. frugiperda in North America ${ }^{9}$. Analysis of $S$. frugiperda lures from North American and English sources yielded Z9-14Ac and Z11$16 A c$ in ratios varying from $80: 20$ to $85: 15$. This result was surprising since addition of Z11-16Ac and Z9-14Ac 
did not increase attraction to Z9-12Ac ${ }^{10}$. The observation of Z9-14Ac, Z11-16Ac and Z9-12Ac in S. frugiperda from Guadeloupe (cited as a personal communication from C. Descoins) ${ }^{10}$ suggested an examination of the antennally active components (GC/EAD) in the pheromone gland of $S$. frugiperda from Costa Rica could be beneficial. This analysis revealed Z9-14Ac, Z11-16Ac and Z7-12Ac as the major antennally active pheromone gland extract components ( $R$. Gries, unpublished). The above suggested that we should concentrate on Z9-14Ac, Z11-16Ac, Z7-12Ac and Z9-12Ac in the development of a lure for $S$. frugiperda in our region. A test of attraction by these acetates presented singly revealed that only Z7-12Ac and Z9-12Ac significantly attracted $S$. frugiperda males. In North America $S$. frugiperda exhibit significant attraction to Z9-14Ac and Z9-12Ac when these acetates are presented singly ${ }^{11}$.

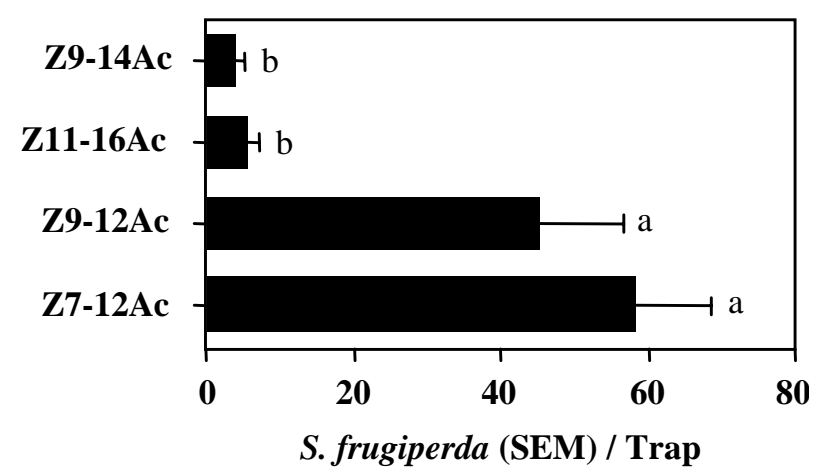

Figure 1: Test of Z9-14Ac, Z11-16Ac, Z7-12Ac and Z9-12Ac as single component attractants for $S$. frugiperda. Test conducted February 12-26, 1998 in Costa Rica. Insects were counted and removed from traps every 3 days. ANOVA $(n=10)$ gave $d f=3,24$; $\mathrm{F}=13.07 ; \mathrm{p}<0.05$.
In a test of binary combinations of the four acetates under study attraction of $S$. frugiperda to Z7-12Ac was increased when it was combined with Z9-14Ac but not with Z11-16Ac or Z9-12Ac (Figure 2). The only binary combination of acetates exhibiting significant attraction in this experiment contained both Z9-14Ac and Z7-12Ac. The ratios Z11-16Ac to Z7-12Ac and Z9-14Ac to Z7$12 \mathrm{Ac}$ used in this experiment were chosen to reflect the ratio of Z11-16Ac and Z9-12Ac to Z9-14Ac in the gland extract of $S$. frugiperda from Guadaloupe and the most attractive ratio of Z9-14Ac (only common component to both these blends) to Z7-12Ac used in lures examined by Tumlinson ${ }^{9}$. Compositions of other binary blends are based on the reported compositions of gland extracts of $S$. frugiperda from Guadeloupe and the most attractive blends as determined by field trials for S. frugiperda in North America 9 .

Since North American and English lures for $S$. frugiperda were binary mixtures of acetates we compared these lures with lures containing the best binary blend from Figure 2 and a blend containing Z9-14Ac and Z11$16 \mathrm{Ac}$ that more nearly mimicked the reported ratio of these components in female pheromone gland extracts of $S$. frugiperda in North America than did the commercial lures (Figure 3). This test revealed that the binary blend of Z914Ac and Z7-12Ac (99.6: 0.4) captured $~ 10 \mathrm{X}$ more male $S$. frugiperda than other binary combinations tested.

To determine if additional components would increase attraction to the most attractive blend from previous experiments (Z9-14Ac : Z7-12Ac, $99.6: 0.4$ ) this blend was tested against blends prepared by adding either Z11$16 \mathrm{Ac}$ or Z9-12Ac alone or in combination to this blend

\author{
Z9-14Ac (0) : Z11-16Ac (96.2) : Z7-12Ac (3.8) : Z9-12Ac (0) \\ Z9-14Ac (0) : Z11-16Ac (33.3) : Z7-12Ac (0) : Z9-12Ac (66.7) \\ Z9-14Ac (0) : Z11-16Ac (0) : Z7-12Ac (2.1) : Z9-12Ac (97.9) \\ Z9-14Ac (0) : Z11-16Ac (0) : Z7-12Ac (0) : Z9-12Ac (100) \\ Z9-14Ac (77.8) : Z11-16Ac (0) : Z7-12Ac (0) : Z9-12Ac (22.2) \\ Z9-14Ac (87.50) : Z11-16Ac (12.5) : Z7-12Ac (0) : Z9-12Ac (0) \\ Z9-14Ac (99.4) : Z11-16Ac (0) : Z7-12Ac (0.6) : Z9-12Ac (0)
}

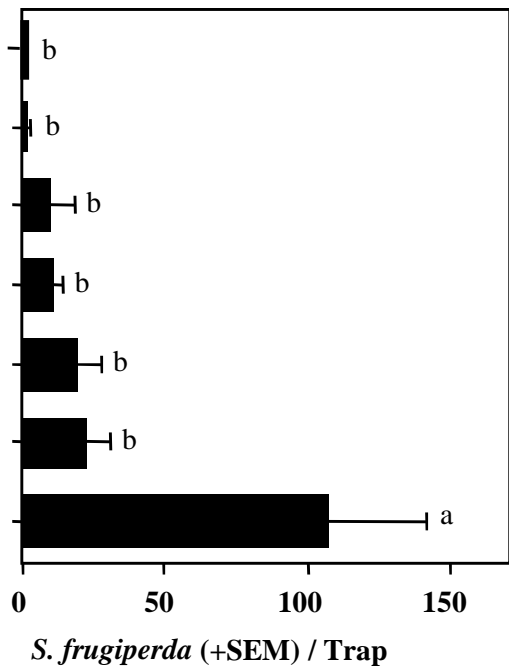

Figure 2: Test of binary combinations of Z9-14Ac, Z11-16Ac, Z7-12Ac and Z9-12Ac vs Z7-12Ac as attractants for S. frugiperda. Test conducted January 3-22, 1999 in Costa Rica. Insects were counted and removed from traps every week. ANOVA $(\mathrm{n}=10)$ gave df $=6,62 ; \mathrm{F}=6.82$; $\mathrm{p}<0.05$. 
Z9-14Ac (97.5): Z11-16Ac (2.5) : Z7-12Ac (0) : Z9-12Ac (0)

Z9-14Ac (80) : Z11-16Ac (20) : Z7-12Ac (0) : Z9-12Ac (0) Engl

Z9-14Ac (85) : Z11-16Ac (15) : Z7-12Ac (0) : Z9-12Ac (0) N A

Z9-14Ac (99.6) : Z11-16Ac (0) : Z7-12Ac (0.4) : Z9-12Ac (0)

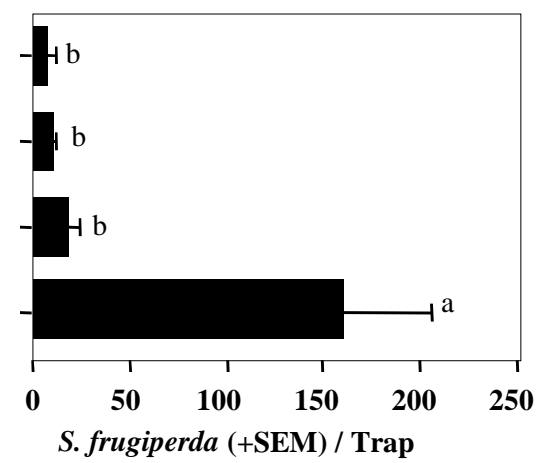

Figure 3: Comparison of North American, English and Costa Rican formulations for S. frugiperda. Test conducted September 22-30, 1997 in Costa Rica. Insects counted and removed every 3-4 days. ANOVA $(\mathrm{n}=10)$ gave df $=3,36 ; \mathrm{F}=9.9 ; \mathrm{p}<0.05$.

\author{
Z9-14Ac (70) : Z11-16Ac (10) : Z7-12Ac (0) : Z9-12Ac (20) \\ Z9-14Ac (77.4) : Z11-16Ac (0) : Z7-12Ac (0.47) : Z9-12Ac (22.1) \\ Z9-14Ac (69.7) : Z11-16Ac (9.96) : Z7-12Ac (0.42) : Z9-12Ac (19.9) \\ Z9-14Ac (99.4) : Z11-16Ac (0) : Z7-12Ac (0.6) : Z9-12Ac (0) \\ Z9-14Ac (87) : Z11-16Ac (12.5) : Z7-12Ac (0.5) : Z9-12Ac (0)
}

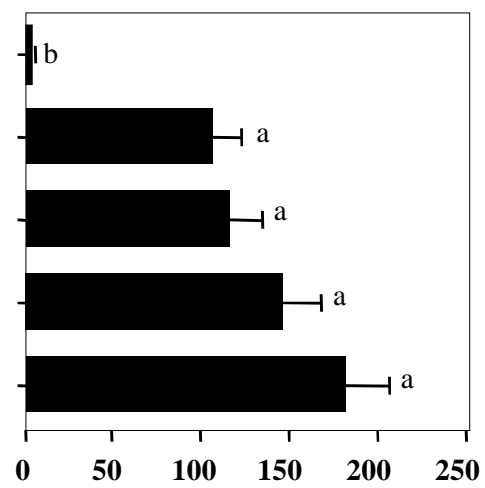

S. frugiperda (+SEM) / Trap

Figure 4: Test of ternary and quaternary blends of Z9-14Ac, Z11-16Ac, Z7-12Ac and Z9-12Ac as attractants for S. frugiperda. Tests conducted October 6-30, 1997 in Costa Rica. Five replicates at one location, ten replicates at a second location and 10 replicates at a third location. Insects counted and removed every 3-4 days. ANOVA gave no location effects so locations were combined. ANOVA on combined data gave on $\log (\mathrm{X}+0.5)$ transformed data, $\mathrm{F}=79.96, \mathrm{df}=4,120, \mathrm{p}<0.05$. Means presented un-transformed.

Z9-14Ac (85) : Z11-16Ac (10.0) : Z7-12Ac (5.0) : Z9-12Ac (0)

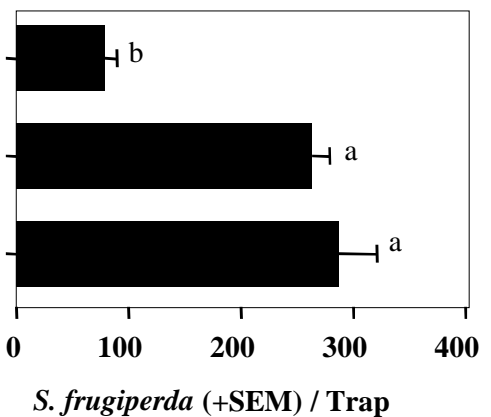

Figure 5: Test of ternary mixtures of Z9-14Ac, Z11-16Ac, Z7-12Ac and Z9-12Ac as attractants for S. frugiperda in which proportion of Z7$12 \mathrm{Ac}$ is increased. Test conducted March 27 to April 13, 1999 in Costa Rica. Insects were counted and removed from traps every week. ANOVA $(\mathrm{n}=10)$ gave $\mathrm{df}=2,27 ; \mathrm{F}=25.24 ; \mathrm{p}<0.05$

(Figure 4). In this experiment the amounts of Z11-16Ac or Z9-12Ac added to the blend of Z9-14Ac and Z7-12Ac were chosen to reflect either the composition of gland extracts of $S$. frugiperda from Guadeloupe or attractive blends for $S$. frugiperda in North America. An additional treatment in Figure 4 was used to examine the possibility of replacement of Z7-12Ac by Z11-16Ac and Z9-12Ac. This alteration completely destroyed the activity of the blend. Addition of Z11-16Ac or Z9-12Ac to the previously attractive blend of Z9-14Ac and Z7-12Ac did not significantly increase attraction. However, it was noted that in the three trials undertaken in this experiment that numerically higher captures were consistently obtained to blends of Z9-14Ac, Z11-16Ac and Z7-12Ac than to the binary blend of Z9-14Ac and Z7-12Ac. Since our goal was development of a mass trapping lure we adopted the numerically superior lure from Figure 4 for an additional optimization. 
Since Z7-12Ac was an essential and minor component of the numerically superior lure in Figure 4 we conducted an experiment to determine the sensitivity of $S$. frugiperda to the amount Z7-12Ac present in the ternary blend of Z914Ac, Z11-16Ac and Z7-12Ac (Figure 5). Increasing the concentration of $\mathrm{Z} 7-12 \mathrm{Ac}$ from $0.5 \%$ to $2 \%$ in the ternary blend did not significantly alter attraction whereas increasing the concentration of Z7-12Ac to $5 \%$ significantly decreased attraction.

The composition of the lure emerging from this study is similar one of the optimal lures deduced for $S$. frugiperda in North America that contains Z9-14Ac and Z7-12Ac (99.4 : 0.6). ${ }^{9}$ Our optimized lure is significantly different in composition than the commercial lures available from North America and England that contain Z9-14Ac and Z11$16 \mathrm{Ac}(\sim 8: 2)$. Current commercial lures produced and used in Costa Rica utilize the most attractive ternary blends of Z9-14Ac, Z11-16Ac and Z7-12Ac shown in Figure 5. These lures are also significantly attractive to $S$. frugiperda in Brazil (E. Vilela, Unpublished). It is noteworthy that Z$12 \mathrm{Ac}$ is an essential component of the optimized blend but was not detected in $S$. frugiperda from Guadeloupe (cited as a personal communication from C. Descoins) ${ }^{10}$.

\section{Acknowledgements}

We wish to thank the technical staff at DelMonte Fresh Produce of Costa Rica for excellent field assistance.

\section{References}

1. Sparks, A. Fla. Entomol. 1979, 62, 82.

2. Lopez, J. D. Jr.; Shaver, T. N.; Goodenough, J. L. J. Chem. Ecol. 1990, 16, 3479.

3. Webber, D. C.; Ferro, D. N. J. Econ. Entomol. 1991 , 84, 1364.

4. Huber, R. T.; Moore, L.; Hoffmann, M. P. J. Econ. Entomol. 1979, 72, 222.

5. Willson, H. R.; Trammel, K. J. Econ. Entomol. 1980, 73, 291.

6. Dunkelblum, E., C.; Rodriguez, C.; Oehlschlager, A. C.; Vargas, M. Manejo Integrado de Plagas, Costa Rica, 1995, 37, 34.

7. Pair, S. D.; Raulston, J. R.; Westbrook, J. K.; Wolf, W. W.; Adams, S. D. Fla. Entomol. 1989, 74, 200.

8. Mitchell, E. R.; McNeil, J. N.; Westbrook, J. K.; Silvain, J. F.; Lalanne-Cassou, B., Chalfant, R. B.; Pair, S. D.; Waddill, V. H.; Sotomayor-Rios, A.; Proshold, F. I. J. Entomol. Sci.; 1991, 26, 39.

9. Tumlinson, J. H.; Mitchell, E. R.; Teal, P. E.; Heath. R. R.; Mengelkoch, L. J. J. Chem. Ecol., 1986, 12, 1901.

10. Mitchell, E. R.; Sugie, H.; Tumlinson, J. H. J. Environ. Sci. Health 1983, A18, 463.

11. Mitchell, E. R.; Doolittle, R. E. J. Econ. Entomol. 1976, 69, 324.

12. Mitchell, E. R.; Copeland, W. W.; Sparks, A. N.; Sekul, A. A. Environ. Entomol. 1974, 3, 778. 